\title{
Rendimiento reproductivo de hembras de Cryphiops caementarius (Crustacea: Palaemonidae) mantenidas con alimento natural
}

\section{Reproductive Performance of female of Cryphiops caementarius (Crustacea: Palaemonidae) maintained with natural food}

\author{
Magali Bazán', Silvia Gámez y Walter Eduardo Reyes²
}

\begin{abstract}
1 Escuela de Biología en Acuicultura. Universidad Nacional del Santa.

2 Departamento de Biología, Microbiología y Biotecnología. Facultad de Ciencias. Universidad Nacional del Santa. Av. Universitaria s/n Urb. Bellamar. Nvo. Chimbote. Ancash. Perú. Email Walter Reyes: wreyes@uns.edu.pe
\end{abstract}

\begin{abstract}
Resumen
El objetivo del presente trabajo fue determinar el rendimiento reproductivo de hembras de C. caementarius mantenidas con alimento natural. Se empleó 24 hembras inmaduras $(5,2 \mathrm{~cm}$ y $2,0 \mathrm{~g})$, acondicionadas en ocho acuarios ( $45 \mathrm{~L}$ ) y alimentadas durante dos meses de acuerdo a cada tratamiento, con pota (Dosidicus sp.), almeja (Semele solida), poliqueto (Pseudonereis sp.) y con alimento balanceado. El rendimiento reproductivo de las hembras fue mejorado cuando se alimentó con poliqueto y pota, lográndose la maduración entre 16 y 18 días con alta fecundidad (2627 y 1377 huevos g $^{-1}$ ) y fertilidad (2566 y 1364 larvas g $^{-1}$, respectivamente).
\end{abstract}

Palabras Claves: Camarón, Cryphiops caementarius, nutrición, reproducción.

\section{Abstract}

The aim was to determine the reproductive performance of females of $C$. caementarius maintained with natural food. Twenty four females inmature were used $(5,2 \mathrm{~cm}$ and $2,0 \mathrm{~g})$, conditioned in eight aquarium (50 I) and fed during two months according to each treatment, with giant squid (Dosidicus sp), clam (Semele solid), polychaete (Pseudonereis sp.) and with balanced. The reproductive performance of females was improved when fed with polychaete and giant squid, achieving maturation between 16 to 18 days with higher fecundity (2627 and 1378

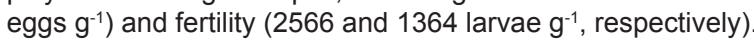

Keywords: Prawn, Cryphiops caementarius, nutrition, reproduction.

\section{Introducción}

Cryphiops caementarius (Molina 1872), habita en los ríos del centro y sur peruano donde tiene importancia económica. Es ovíparo, unisexual con fertilización externa cuya talla de primera madurez sexual es de $33 \mathrm{~mm}$ de longitud total en hembras y 22,5 mm en machos (Lip 1976); además el cortejo, apareamiento y desove se realizan sin inconvenientes en cautiverio. En su ambiente natural $C$. caementarius se alimenta de detritus, microalgas (Viacava et al. 1978) y en cautiverio acepta todo tipo de alimento fresco y balanceado; además, suele alimentarse en la noche y es no selectivo.

La nutrición es importante en la maduración de crustáceos (Cahu 1998). En peneidos, se emplean dietas secas, con calamar, ostras (Gómez y Arellano 1987), almejas (Millanema y Quinito 2000), poliquetos (Wouters et al. 2001), logrando incrementar la frecuencia de maduración, el número de huevos y larvas por desove y la eclosión. El empleo de estos tipos de alimento no se ha investigado en $C$. caementarius.

El alimento natural promueve la maduración y el desove en crustáceos, por los elevados niveles de proteínas y de ácidos grasos esenciales para vitelogénesis (Xu et al. 1994), además eleva la fecundidad y fertilidad (Cahu et al. 1998). En Dosidicus gigas, el contenido proteico y de lípidos del manto de es de 85,35 y 2,31\% respectivamente (Martínez-Vega et al. 2000) mientras que en la almeja Gary solida de 27,71 y 1,65\% (Bear 1998). Los poliquetos en la dieta proveen de nutrientes (Kawahigashi 1998) y principalmente de hormonas que estimulan la maduración (Wouters et al. 2001).

El objetivo del presente estudio fue determinar el rendimiento reproductivo de hembras del camarón de río $C$. caementarius mantenidas con alimento natural, constituido por pota (Dosidicus sp.), almeja (Semele solida) y poliqueto (Pseudonereis sp.), en condiciones de laboratorio.

\section{Material y métodos}

Los camarones fueron capturados en el río Lacramarca (0907'70”S y 78³4’20”W), provincia del Santa, departamento de Ancash. En el laboratorio los ejemplares de C. caementarius (Molina 1872) fueron identificados (Méndez 1982) y luego aclimatados en acuarios durante una semana. Fueron seleccionadas 24 hembras con ovarios en estado II (Viacava et al. 1978) las que tuvieron 5,2 $\pm 1,2 \mathrm{~cm}$ de longitud total (LT) y 2,0 $\pm 0,2 \mathrm{~g}$ de peso total (PT). Así mismo, se seleccionaron camarones machos de $5,8 \pm 0,1 \mathrm{~cm}$ LT y $3,1 \pm 0,2 \mathrm{~g}$ PT. Se emplearon ocho acuarios (45 L), cada uno con tres divisiones, lo que permitió cuatro tratamientos, constituyendo cada hembra una repetición.

Las hembras fueron alimentadas durante dos meses, con carne de mantos de pota (Dosidicus sp.), carne de almeja (Semele solida), poliqueto (Pseudonereis sp.) y con alimento balanceado comercial para reproductores de camarón de mar $(40 \%$ de proteína total y $5 \%$ de grasas totales). La pota y la almeja fueron adquiridas del mercado La Sirena de Chimbote; en cambio los poliquetos fueron recolectados de las zonas rocosas de la Playa El Dorado de la bahía Samanco (Ancash).

El alimento natural fue analizado según la metodología de AOAC (2005). Se encontró que los poliquetos contenían 11,1 y $1,8 \%$ de proteína y grasas totales respectivamente; mientras que pota 15,3 y $0,6 \%$ y almeja 14,4 y $0,5 \%$. El alimento natural, fue lavado, cortado en trozos de $5 \mathrm{~mm}$, envueltos en papel de aluminio y congelados $\left(-1^{\circ} \mathrm{C}\right)$ hasta por tres días. Los camarones fueron alimentados a las 08:00 y 18:00 horas, con el 30 y 70\% del alimento respectivamente y que correspondió al 15\% del peso de cada camarón.

Para obtener hembras ovíferas con huevos viables se juntó a una hembra con ovario en estado IV (Viacava et al. 1978) con un camarón macho en intermuda (Reyes y Lujan 2003). En una muestra de 20 huevos recién puestos fueron medidos el eje mayor 
(L) y menor (l) en un microscopio compuesto equipado con ocular micrométrico (EM-15x Lomo) y con ello se determinó el volumen de los huevos según la fórmula de un elipsoide:

$\left.\mathrm{V}=\pi_{*} \mathrm{~L}_{*} 1^{2} / 6\right)$

La fecundidad $(\mathrm{F})$ se determinó por:

$\left.\mathrm{F}=\left[(\mathrm{Wmo})^{*}(\mathrm{Nh}) / \mathrm{Wh}\right)\right]$

Donde $\mathrm{Wmo}=$ es el peso de la masa ovígera,

$\mathrm{Nh}=$ es el número de huevos en la muestra y

$\mathrm{Wh}=$ es el peso de la muestra de huevos.

Wmo $=($ Wo - Wno $)$, donde Wo es el peso de la hembra con huevos y Wno es el peso de la hembra sin huevos.

La fertilidad (f) se determinó por:

$\mathrm{f}=[(\mathrm{Vr}-\mathrm{Nl}) / \mathrm{Vm}]$,

Donde $\mathrm{Vr}$ es el volumen del recipiente, $\mathrm{Nl}$ es el número de larvas en la muestra y $\mathrm{Vm}$ es el volumen de agua de la muestra.

El 50\% del agua de los acuarios fue renovada semanalmente. Diariamente se realizó limpieza de los acuarios. El oxígeno y la temperatura del agua fueron medidos con oxímetro digital YSI 55 ( $\left.\pm 0,01 \mathrm{mg} \mathrm{L}^{-1} ; 0,01^{\circ} \mathrm{C}\right)$, el $\mathrm{pH}$ con $\mathrm{pH}$-metro digital ( $\pm 0,01$ unidades), la dureza total y el $\mathrm{CO}_{2}$ según Fukushima et al. (1982).

Los resultados fueron sometidos a análisis de varianza de una vía y se realizó comparación de medias con la prueba de Tukey con un nivel de significancia del 1\%. Los análisis estadísticos se efectuaron con el Software SPSS versión 15 para Windows.

\section{Resultados}

Las hembras de $C$. caementarius que consumieron poliqueto y pota maduraron en $16,2 \pm 1,1$ días y $18,0 \pm 1,5$ días, respectivamente, siendo estadísticamente iguales; en cambio las alimentadas con almeja y con balanceado maduraron en 32,4

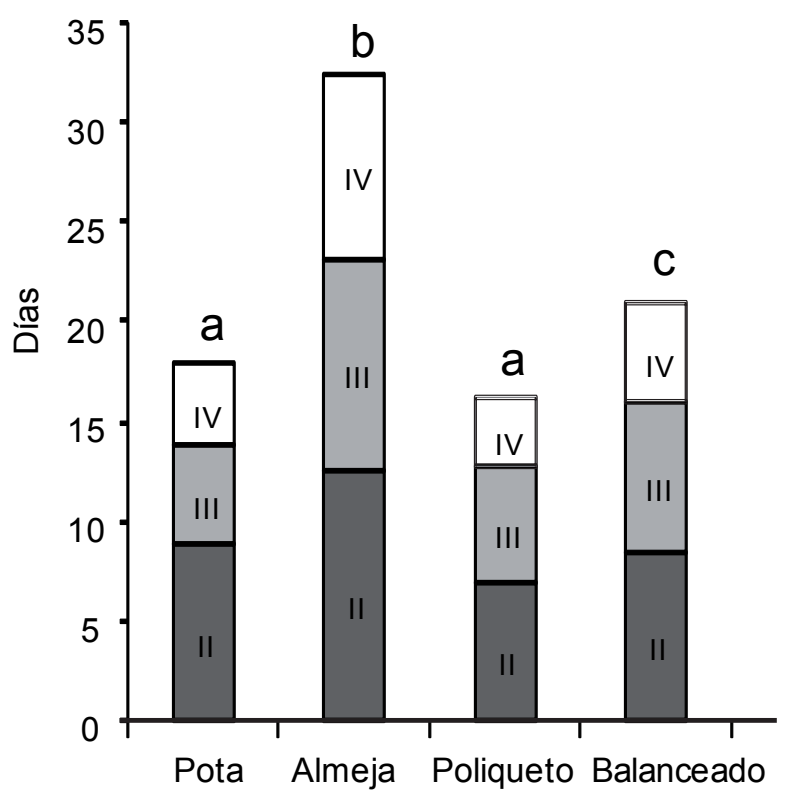

Figura 1. Duración de la maduración de cada estado del ovario (en números romanos) de Cryphiops caementarius alimentados con diferentes tipos de alimento. Columnas con letras iguales indica que no hay diferencia estadística significativa $(p>0,01)$.
Tabla 1. Valores promedios ( \pm desviación estándar) de los ejes mayor $(\mathrm{mm})$ y menor $(\mathrm{mm})$ y del volumen $\left(\mathrm{mm}^{3}\right)$ de los huevos recién puestos de Cryphiops caementarius alimentados con diferentes tipos de alimento.

\begin{tabular}{ccccc}
\hline \multirow{2}{*}{ Medidas } & Pota & Almeja & Poliqueto & Balanceado \\
\hline \multirow{2}{*}{ Eje mayor } & $\begin{array}{c}0,615^{\mathrm{a}} \\
( \pm 0,020)\end{array}$ & $\begin{array}{c}0,590^{\mathrm{a}} \\
( \pm 0,018)\end{array}$ & $\begin{array}{c}0,620^{\mathrm{a}} \\
( \pm 0,022)\end{array}$ & $\begin{array}{c}0,605^{\mathrm{a}} \\
( \pm 0,018)\end{array}$ \\
\hline Eje menor & $\begin{array}{c}0,430^{\mathrm{a}} \\
( \pm 0,025)\end{array}$ & $\begin{array}{c}0,400^{\mathrm{a}} \\
( \pm 0,014)\end{array}$ & $\begin{array}{c}0,460^{\mathrm{a}} \\
( \pm 0,020)\end{array}$ & $\begin{array}{c}0,420^{\mathrm{a}} \\
( \pm 0,015)\end{array}$ \\
\hline Volumen & 0,059 & 0,049 & 0,069 & 0,056 \\
\hline
\end{tabular}

Valores con iguales letras en superíndice en una misma fila indica que no hay diferencia estadística significativa $(p>0,01)$.

$\pm 2,3$ días y 21,0 $\pm 1,3$ días, respectivamente. Además, hubo retraso de cada estado del ovario en las hembras alimentadas con almeja y balanceado; y aceleración con poliqueto y pota siendo similares estadísticamente ( $\mathrm{p}>0,01)$ (Fig. 1).

La fecundidad de las hembras de $C$. caementarius fue significativamente diferente $(\mathrm{p}<0,01)$ entre tratamientos; siendo mayor con poliqueto $\left(2627 \pm 284\right.$ huevos $\left.^{-1}\right)$, seguido por pota (1377 \pm 253 huevos $\left.\mathrm{g}^{-1}\right)$, balanceado $\left(924 \pm 247\right.$ huevos $\left.\mathrm{g}^{-1}\right)$ y almeja $\left(355 \pm 60\right.$ huevos $\left.\mathrm{g}^{-1}\right)$. De igual manera, la fertilidad fue mayor con poliqueto $\left(2566 \pm 320\right.$ larvas g $\left.^{-1}\right)$ y pota $(1364 \pm 252$ larvas $\left.\mathrm{g}^{-1}\right)$ que con balanceado $\left(896 \pm 260\right.$ larvas $\left.\mathrm{g}^{-1}\right)$ y almeja $(333 \pm$ 62 larvas g $^{-1}$ ) (Fig. 2).

No existió diferencias significativas ( $p>0,01)$ en el volumen de los huevos, siendo en promedio el eje mayor de $0,608 \mathrm{~mm}$, el eje menor de $0,428 \mathrm{~mm}$ y el volumen de $0,058 \mathrm{~mm}^{3}$ (Tabla 1 ).

Las hembras de C. caementarius alimentadas con poliqueto, pota y balanceado capturaron rápidamente su alimento, sucediendo lo contrario con almeja. Pocos desechos fecales fueron observados en aquellos camarones alimentados con pota, almeja y poliqueto. 
La concentración de oxígeno $\left(4,52 \pm 1,24 \mathrm{mg} \mathrm{L}^{-1}\right)$, la temperatura $\left(22,55 \pm 0,85^{\circ} \mathrm{C}\right)$, el $\mathrm{pH}(7,05 \pm 0,54)$ y la dureza total $\left(160 \pm 2,85 \mathrm{mg} \mathrm{L}^{-1}\right)$ no fueron estadísticamente diferentes ( $p>0,01)$ entre tratamientos. No se observaron valores anómalos de $\mathrm{CO}_{2}$ en el agua de todos los acuarios.

\section{Discusión}

La rápida maduración ovárica en las hembras de $C$. caementarius alimentadas con poliqueto (16 días) y pota (18 días), en relación con almeja (32 días) y balanceado (21 días), sugiere que ciertos componentes nutricionales y hormonales contenidos en poliqueto y pota aceleraron la maduración. Se conoce que pota suministra altos niveles de proteínas (Martínez-Vega et al. 2000) y los poliquetos proveen hormonas sexuales (Gómez y Arellano 1987; Kawahigashi 1998) que son responsables de la maduración ovárica de Uca vocans borealis (Shih y Tseng 1999). Además, Harrison (1997) considera que los ácidos grasos esenciales, los fosfolípidos y esteroles son importantes porque los crustáceos tienen limitaciones para sintetizarlos.

De igual manera, el mayor número de huevos (2627 huevos $\mathrm{g}^{-1}$ ) y de larvas (2566 larvas $\mathrm{g}^{-1}$ ) fue obtenido en las hembras alimentadas con poliqueto; seguida por pota (1377 huevos $\mathrm{g}^{-1}$

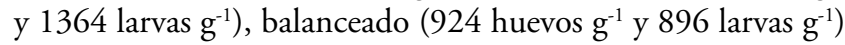
y almeja (355 huevos $\mathrm{g}^{-1}$ y 333 larvas $\mathrm{g}^{-1}$ ). Esto demuestra que poliqueto y pota no solamente poseen nutrientes para acelerar la maduración ovárica sino también para mejorar la fecundidad y la fertilidad de C. caementarius. En Penaeus vannamei alimentado con poliqueto y calamar, se observó incremento en el número de huevos y el numero de larvas, en comparación con la dieta balanceada (Hidalgo 1997), similares resultados también son reportados para Scylla serrata (Millanema y Quinito 2000).

Aunque el tamaño de los huevos de las hembras fueron similares entre tratamientos, sin embargo, se observó tendencia a ser mayor en aquellas alimentadas con poliqueto y pota, lo que sugiere una mejor asimilación y deposición de nutrientes, pues se ha proporcionado evidencia de la transferencia de nutrientes para la conformación de vitelo, desde el intestino medio hasta el ovario durante la maduración ovárica de P. semisulcatus y los ovocitos incrementan de tamaño (Ravid et al. 1999).

Podemos concluir que el rendimiento reproductivo de las hembras de $C$. caementarius fue mejorado cuando se alimentó con poliqueto Pseudonereis sp. y pota Dosidicus sp., lográndose rápida maduración (16 a 18 días), mayor fecundidad (2627 y 1377 huevos $\mathrm{g}^{-1}$ ) y fertilidad (2566 y 1364 larvas $\mathrm{g}^{-1}$, respectivamente).

\section{Literatura citada}

AOAC. 2005. Official Methods of Analysis of AOAC INTERNATIONAL, 18th Ed., AOAC INTERNATIONAL, Gaithersburg, MD.

Beard J. 1998. What is the nutritional value of clams? Comp. of the Interstate Shellfish Sanitation Conference, 4: 216-317.

Cahu C.L. 1998. Diets of shrimp broodstock and theis effect on larval quality. IV Simp. Aquatic. Nutrition, 15-18 Nov. La Paz, BCS. México.

Fukushima M., J. Reyes, G. Sifuentes, et al. 1982. Manual de Métodos Limnológicos. Universidad Nacional de Trujillo, Perú. $120 \mathrm{p}$

Gómez L. \& E. Arellano. 1987. Maduración en cautiverio de Penaeus vannamei en la Escuela Superior Politécnica del Litoral (ESPOL). Tecnológica, 7 (3): 31-41.
Harrison K.E. 1997. Broodstock nutrition and maturation diets. The World Aquaculture Society, 6: 390-408.

Hidalgo M. 1997. Efecto de la composición nutricional de Artemia enriquecida en la reproducción de Penaeus vannamei. Tesis de grado. Escuela Superior Politécnica del Litoral. Facultad de Ingeniería Marítima y Ciencias del Mar. 22 pp.

Kawahigashi D.K. 1998. Overview of commercial maturation technology in the Western hemisphere. pp.381-392. In: Recife Brazil LAC WAS Conference Proceedings. Anais de aquicultura Brazil.1998.

Lip L.G. 1976. Primera madurez sexual del camarón de río Cryphiops caementarius (Molina, 1872) (Natantia Palaemonidae) en el río Moche. Tesis de Bachiller en Ciencias Biológicas. Universidad Nacional de Trujillo. Perú. 79 pp.

Martínez-Vega J., L.E. Cruz-Suárez \& D. Ricque-Marie. 2000. Evaluación de las diferentes partes corporales del calamar gigante (Dosidicus gigas) en forma de harina, en dietas balanceadas para camarón (Litopenaeus vannamei). Ciencia y Mar, 11: 11-17.

Méndez A. 1982. Claves de identificación y distribución de los langostinos y camarones (Crustacea, Decapoda) del mar y ríos de la costa del Perú. Bol. Inst. Mar Perú, 18 (5):1-30

Millanema O.M. \& E. Quinito. 2000. The effects of diets on reproductive performance of eyestalk ablated and intact mud crab Scylla serrata. Aquaculture, 181: 81-90.

Ravid T., A. Tietz, M. Khayat, et al. 1999. Lipid accumulation in the ovarios of marine shrimp Penaeus semisulcatus (De Haan). J. Exp. Biol., 202 (13): 1819-1829.

Reyes W.E. \& H. Lujan. 2003. Estados y subestados del ciclo de muda del camarón de río (Cryphiops caementarius Molina, 1872) (Crustacea, Decapoda, Palaemonidae). En: II Congreso Iberoamericano Virtual de Acuicultura: 808-817. http://www.revistaaquatic.com/civa2003/coms/completo. asp?cod=111 [acceso noviembre 22, 2008].

Shih J.T. \& S.S. Tseng. 1999. Progesterone-like substance in ovary and hepatopancreas of Uca vocans borealis. Zoological Studie, 38 (4): 458-465.

Viacava M., R. Aitken \& J. Llanos. 1978. Estudio del camarón de río en el Perú. 1975-1976. Bol. Inst. Mar Perú, 3 (35): 161-232.

Wouters R., P. Lavens, J. Nieto, et al. 2001. Penaid shrimp broodstock nutrition: an updated review on research and development. Aquaculture, 202: 1-21.

Xu X., W. Li, J. Castell, et al. 1994. Influence of dietary lipid sources on fecundity, egg hatchability and fatty acid composition of Chinese prawn (Penaeus chinensis) broodstock. Aquaculture, 119: 359-370. 


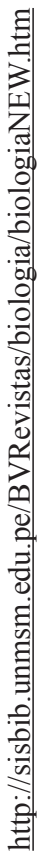

